Article

\title{
A Clip Domain Serine Protease Involved in Egg Production in Nilaparvata lugens: Expression Patterns and RNA Interference
}

\author{
Jia-min Wu, Rong-er Zheng, Rui-juan Zhang, Jin-liang Ji, Xiao-ping Yu * and Yi-peng $\mathrm{Xu}$ *(1) \\ Zhejiang Provincial Key Laboratory of Biometrology and Inspection \& Quarantine, China Jiliang University, \\ Hangzhou 310018, China; wujiamin94@163.com (J.-m.W.); qq651047935@163.com (R.-e.Z.); \\ 18893781226@163.com (R.-j.Z.); j1793505637@163.com (J.-1.J.) \\ * Correspondence: yxp@cjlu.edu.cn (X.-p.Y.); ypxu2002@163.com (Y.-p.X.); Tel.: +86-0571-86835700 (Y.-p.X.)
}

Received: 16 October 2019; Accepted: 29 October 2019; Published: 30 October 2019

\begin{abstract}
Clip domain serine proteases play vital roles in various innate immune functions and in embryonic development. Nilaparvata lugens proclotting enzymes (NlPCEs) belong to this protease family. NIPCE1 was reported to be involved in innate immunity, whereas the role of other NIPCES is unclear. In the present study, N. lugens proclotting enzyme-3 (NIPCE3) was cloned and characterized. NIPCE3 contains a signal peptide, a clip domain, and a trypsin-like serine protease domain. NIPCE3 was expressed in all tissues examined (gut, fat body, and ovary), and at all developmental stages. Immunofluorescence staining showed that NIPCE3 was mainly expressed in the cytoplasm and cytomembrane of follicular cells. Double stranded NIPCE3 RNA interference clearly inhibited the expression of NIPCE3, resulting in abnormal egg formation and obstruction of ovulation. These results indicate that NIPCE3 plays an important role in egg production in N. lugens.
\end{abstract}

Keywords: Nilaparvata lugens; proclotting enzyme; RNAi; follicular cell; egg production

\section{Introduction}

The brown planthopper (BPH), Nilaparvata lugens, is one of the most harmful rice crop pest insects throughout Asia and causes tremendous economic losses upon emergence [1]. N. lugens easily develops resistance to pest-resistant rice varieties and insecticides, making it hard to effectively control. To a large extent, this is ascribed to $N$. lugens being an R-selected species with high fertility and strong invasive capacity [2]. Therefore, genes involved in ovarian development or oogenesis are attractive targets for controlling $N$. lugens through interfering with reproduction [3,4].

Based on an analysis of genome and transcriptome data, there are five proclotting enzyme (PCE) genes in N. lugens genome [5]. PCEs belong to the clip-domain serine protease (clip-SPs) family, which play vital roles in embryonic development and various innate immune functions in invertebrates such as antimicrobial activity, cell adhesion, hemolymph clotting, pattern recognition, and regulation of the prophenoloxidase system [6]. PCE in the horseshoe crab Tachypleus tridentatus was the first serine protease-containing clip domain protein identified, and the domain was named such because it can be drawn in the shape of a paper clip in a schematic form to show the disulfide linkages $[7,8]$. In T. tridentatus, PCE is involved in a coagulation cascade that has since been widely employed as a simple and very sensitive assay for endotoxins [9]. Clip-SPs have also been reported from many species, such as immune response in Chlamys farreri [10], antibacterial defense in Penaeus monodon [11], prophenoloxidase activation cascade in Manduca sexta [12-14], Aedes aegypti [15], Anopheles gambiae [16,17], Tenebrio molitor [18], Holotrichia diomphalia [19,20], Bombyx mori [21], and the Toll signaling pathway on dorsal-ventral axis of Drosophila melanogaster embryogenesis [22-25]. However, the functions of most clip-SPs are unknown, even in well studied insect species [23]. 
In N. lugens, NlPCEs were considered to mediate innate immunity [5], where NlPCE1 showed bacteria-induced gene expression [26]. NIPCE1 therefore appears to have a role in the host defense response, but the function of other PCEs in N. lugens is still unclear. In our previous study of N. lugens ovarian transcriptome (data unpublished), we found NIPCE3 was highly expressed in the ovary, which implied NIPCE3 to be important in ovarian development. In the present study, NIPCE3 was cloned and its encoded protein sequence was analysed. RNA interference (RNAi) was then used to knock down its expression, and its functions in N. lugens were also investigated.

\section{Materials and Methods}

\subsection{Insect Rearing}

$N$. lugens used in this study were maintained in a climatron at China Jiliang University, Hangzhou, China. The insects were reared on rice seedlings (Taichung Native 1, TN1) at $27 \pm 1{ }^{\circ} \mathrm{C}$ with $60 \%$ humidity under a $16 \mathrm{~h}$ light: $8 \mathrm{~h}$ dark photoperiod.

\subsection{Cloning the CDNA of NIPCE3}

Total RNA was isolated from $N$. lugens adult females with a TaKaRa MiniBEST Universal RNA Extraction Kit (Takara, Dalian, China), according to the manufacturer's instructions. The RNA quantity was confirmed by agarose gel electrophoresis and analysed with a NanoDrop 2000 spectrophotometer (Thermo Scientific, Waltham, MA, USA). One $\mu g$ total RNA was then used to synthesize cDNA in $20 \mu \mathrm{L}$ reactions using PrimeScript ${ }^{\mathrm{TM}}$ II 1st Strand cDNA Synthesis Kit (Takara, Dalian, China). In order to clone the NIPCE3 sequence, a PCR primer pair for NIPCE3 was designed with primer premier 5.0 (Table 1). Premix Taq ${ }^{\mathrm{TM}}$ (Takara, Dalian, China) was used to amplify the PCR product. PCR product was cloned into a pMD19-T vector (Takara, Dalian, China) and then sequenced by Sanger's method.

\subsection{Sequence Comparison and Phylogenetic Analysis}

The NlPCE3 protein sequence was compared with other Clip-SP sequences from a NCBI BLAST (https://blast.ncbi.nlm.nih.gov/Blast.cgi), and these sequences were aligned with ClustalW. Physical parameters and signal peptide position were predicted by ExPASy (https://web.expasy.org/protparam/) and SignalP (http://www.cbs.dtu.dk/services/SignalP/), respectively. Transmembrane helices were analysed by TMHMM Server v. 2.0 (http://www.cbs.dtu.dk/services/TMHMM-2.0/). Phosphorylated sites were predicted by KinasePhos (http://kinasephos.mbc.nctu.edu.tw/predict.php).

\subsection{Real-time Quantitative PCR Analysis}

Real-time quantitative PCR (qPCR) was carried out to analyse the expression level of target genes. Total RNA was isolated as described above. One $\mu \mathrm{g}$ RNA was used for reverse transcription in a $20 \mu \mathrm{L}$ reaction using Perfect Real Time PrimeScript ${ }^{\mathrm{TM}}$ RT reagent Kit with gDNA Eraser (Takara, Dalian, China). qPCR was performed in triplicate on Step One plus (ABI, USA) using SYBRßPremix Ex Taq $^{\mathrm{TM}}$ II (Tli RNaseH Plus) (Takara, Dalian, China). A specific primer pair for qPCR was designed and $N$. lugens $18 S$ rRNA (Nl18S) was used as an internal control (Table 1). The qPCR profile was: $94{ }^{\circ} \mathrm{C}$ for $30 \mathrm{~s}$ followed by 40 cycles at $94{ }^{\circ} \mathrm{C}$ for $5 \mathrm{~s}$ and $60{ }^{\circ} \mathrm{C}$ for $30 \mathrm{~s}$. The specificity of primers was confirmed by melting curve analysis and Sanger sequencing. According to the curve based on the cycle threshold and logarithm of copy concentration, the amplification efficiency of NIPCE3 and NI18S primers were calculated and they are both $103 \%$, so the expression variation of NIPCE3 can be evaluated by $2^{-\Delta \Delta C t}$ method.

\subsection{RNA Interference}

Because the +1 to +6 region of $\mathrm{T} 7$ promoter is vital for the efficient synthesis of double strand RNA (dsRNA) in vitro using T7 RNA polymerase and synthetic DNA templates [27], we carefully searched the special six nucleotide bases in target gene sequences, and primers containing the $\mathrm{T} 7$ 
promoter sequence for synthesizing dsRNA were designed (Table 1). The template of NIPCE3 for dsRNA synthesis was $630 \mathrm{bp}$ (from 798 to 1427). dsRNA of GFP gene (dsGFP) was taken as the negative control. The GFP gene sequence was synthesized in vitro referred to the binary vector pCAMBIA-1302 (GenBank: AF234298.1), and was cloned to pMD19-T vector (Takara, Dalian, China). The template of GFP for dsRNA synthesis was $350 \mathrm{bp}$ (from 282 to 631). The PCR amplification product of the target gene was used as the template to synthesize dsRNA using a MEGAscript 77 transcription kit (Ambion, Austin, TX, USA), according to the manufacturer's instructions. The quality and size of the dsRNA products were verified by $1 \%$ agarose gel electrophoresis and the NanoDrop 2000 spectrophotometer. Approximately $50 \mathrm{~nL}$ of dsRNA $(5000 \mathrm{ng} / \mu \mathrm{L})$ was injected into the abdomen of each newly emerged virgin macropterous female using a manual microinjector.

Table 1. The primers used in this study.

\begin{tabular}{ll}
\hline \multicolumn{1}{c}{ Primers } & Primer Sequence $\left(\mathbf{5}^{\prime}-\mathbf{3}^{\prime} \mathbf{)}\right.$ \\
\hline $\begin{array}{l}\text { for cloning cDNA } \\
\text { NlPCE3-F }\end{array}$ & CGGCAGTCTGCTTCAGTTT \\
for qRT-PCR & GTCGAAAACTAGGTCAAACAT \\
NlPCE3-qF & CGAAATGGAAGATTGCTGAGTC \\
NlPCE3-qR & TGGTGTTGGCGTTGATTATGG \\
Nl18S-qF & GTAACCCGCTGAACCTCC \\
Nl18S-qR & GTCCGAAGACCTCACTAAATCA \\
for synthesizing dsRNA & \\
T7-NlPCE3-dsF & GGATCCTAATACGACTCACTATAGGGACTCTCGTTTCCAGATACC \\
T7-NlPCE3-dsR & GGATCCTAATACGACTCACTATAGGCACCAGTGACTTCGCTC \\
T7-GFP-dsF & GGATCCTAATACGACTCACTATAGGGATACGTGCAGGAGAGGAC \\
T7-GFP-dsR & GGATCCTAATACGACTCACTATAGGGCAGATTGTGTGGACAGG \\
\hline
\end{tabular}

\subsection{Dissection Observation and Fertility Analysis}

Insects treated with dsRNA were dissected in PBS. The dissected tissues were observed under a stereozoom microscope (Nikon SMZ1500, Tokyo, Japan), and photographed with NIS Elements software. To analyse fertility, one treated female ( $24 \mathrm{~h}$ after dsRNA treatment) was mated with two untreated males in a bottle containing fresh rice seedlings that were changed every day. Mortalities were calculated each day and the number of eggs was counted after oviposition until the females died.

\subsection{Immunofluorescence}

Immunofluorescence was performed to analyse the localization and distribution of target proteins. The ovary, fat body, and gut were dissected from macropterous females. Fixing and staining were performed as previously described [28]. Anti-NIPCE3 primary rabbit polyclonal antibody was purified from New Zealand rabbits after injection of an NlPCE3 polypeptide (VEGKSRHRRSIGDQ) antigen solution. The specificity of the anti-NlPCE3 antibody was evaluated by western blot (Figure S1). Goat anti-rabbit IgG antibody conjugated with Dylight 488 fluorescent dye was used as a secondary antibody. The samples were observed with a laser scanning confocal microscopy (Leica SP8, Mannheim, Germany).

\section{Results}

\subsection{Identification and Phylogenetic Analysis of NIPCE3}

The ORF of NlPCE3 potentially encodes 460 amino acids (Figure 1) (GenBank: MN586815). The predicted protein NlPCE3 is deduced with a molecular mass of approximately $52.2 \mathrm{kDa}$ and a calculated pI value of 8.94. NIPCE3 contains a 20 amino acid N-terminal signal peptide, but no transmembrane domain. As expected, NIPCE3 does contain two typical clip serine protease domains, 
a clip domain with six cysteines, and a trypsin-like serine protease domain with the three conserved catalytic sites (H264, D317, and S410). NlPCE3 has 13 phosphorylation sites, including seven serine sites (S45, S118, S124, S125, S164, S183, and S201), two threonine sites (T119 and T124) and four tyrosine sites (Y149, Y309, Y345, and Y444).

Clip-SP sequences from several species were aligned with NlPCE3 and compared. The alignment shows that all sequences have two conserved structural domains with six cysteines and three conserved catalytic sites (Figure S2). The two domains of NlPCE3 exhibit high homology with those of other clip-SP sequences. However, the intervening sequence between the clip and serine protease domains in NIPCE3 has low identity to that of other clip-SPs. Sequence comparisons also show that NlPCE3 is 32\%, 29\%, 26\%, and 20\% identical to NlPCE1, NlPCE2, NlPCE4, and NlPCE5, respectively (Figure S3).

1 cggcagtctg cttcagtttc agtgatcagt gatgttgtaa ggccaacATG AGGTTCATTT TGAGCGTCTT CTTCATTGCA TCCACCTTTT $\begin{array}{llllllllllllll}M & R & F & T & I & S & V & F & F & T & A & S & T & F\end{array}$

91 TCGCTTCTTC TCTGTCACAC AGGAATCCAG CCGAAAGGCG AGTGGATGAA CTAAGTCATC GGTTGAGAAG AGCTCTACCA AAAGAGAATT

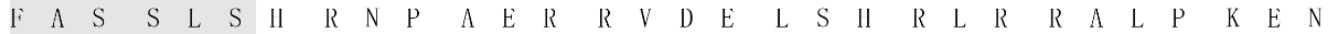

181 CAAAGGAAGC GAATCCAACT TGCTATGGCT ACAATGGCCA GAAAGGCAGA TGCTTGCGTC TTGAAGACTG TTTTTTGCAG CCTTTGAAAG

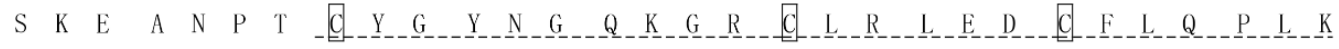

271 AAACTACAT CAAGTACAGA GCTTACTACT GCATCATTGG CAGTGAAGTT GGTGTTTGCT GTCCAAATGC CATTGCTATT CCTGAAAGT E_ N_I I I K Y

361 ACAATGAGAA CCAGTTCCTA CCGGCAGCCA GGAGGAACTC GACAGGAAGG AGGCGTTCTT CAAGATCTGT CACTTACGAA ATGACACCTO

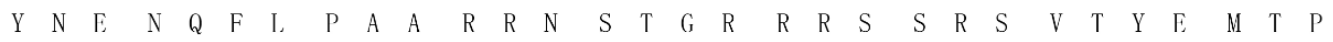

451 CTTATTCAGA AAGCAAGgTT TTAGGTCGTC CAAGTGATGT ATATGAAGAT CAGCAAAGCA AATCGAAATT TCCCAAAAAG TACTCCAGCG

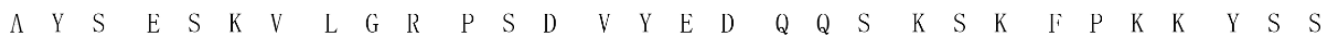

541 ATAATCATGA TAAAAAGAAG ATTCCAAAAA AATATTCCAT CAATAAGAAG AAAAGTATGA AAGAGATGAA CGCCGTAGAG GTTGAAGGGA

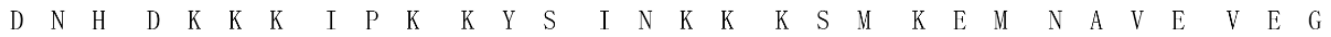

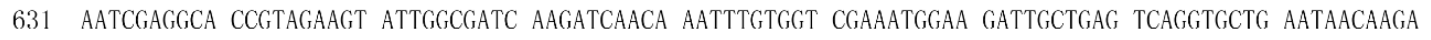

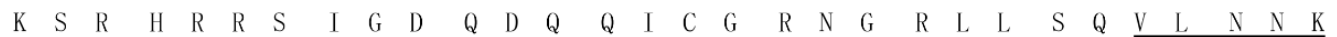

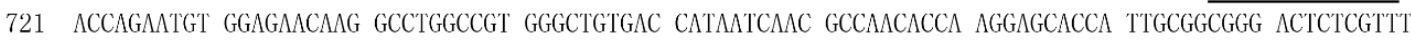
\begin{tabular}{lllllllllllllllllllllllllllllll}
$N$ & $Q$ & $N$ & $V$ & $E$ & $N$ & $K$ & $A$ & $W$ & $P$ & $W$ & $A$ & $V$ & $T$ & $I$ & $I$ & $N$ & $A$ & $N$ & $T$ & $K$ & $E$ & $H$ & $H$ & $C$ & $G$ & $G$ & $T$ & $L$ & $V$ \\
\hline
\end{tabular}

811 CCAGATACCA CGTCATCTCG GCAGCTCACT GTTTCATTAT CGATGGTGGA CACGTGATGA AGGAGGTGAT CTTGGCGCGG ATGGGCGAGT \begin{tabular}{lllllllllllllllllllllllllllllllllll}
$\mathrm{S}$ & $\mathrm{R}$ & $\mathrm{Y}$ & $\mathrm{H}$ & $\mathrm{V}$ & $\mathrm{T}$ & $\mathrm{S}$ & $\mathrm{A}$ & $\mathrm{A}$ & $\mathbb{H}$ & $\mathrm{C}$ & $\mathrm{F}$ & $\mathrm{T}$ & $\mathrm{T}$ & $\mathrm{D}$ & $\mathrm{G}$ & $\mathrm{G}$ & $\mathrm{H}$ & $\mathrm{V}$ & $\mathrm{M}$ & $\mathrm{K}$ & $\mathrm{E}$ & $\mathrm{V}$ & $\mathrm{T}$ & $\mathrm{I}$ & $\mathrm{A}$ & $\mathrm{R}$ & $\mathrm{M}$ & $\mathrm{G}$ & $\mathrm{E}$ \\
\hline
\end{tabular}

901 ACGACTTCAA GCACGACGAC GACAGCCGCT TCTATGACTA CGAGGTGGAG CGCATCATCA ACCACCCTGA CTACGACGAC CCCATCCATG \begin{tabular}{lllllllllllllllllllllllllllllll}
$Y$ & $D$ & $\mathrm{~F}^{\mathrm{i}}$ & $\mathrm{K}$ & $\mathrm{II}$ & $\mathrm{D}$ & $\mathrm{D}$ & $\mathrm{D}$ & $\mathrm{S}$ & $\mathrm{R}$ & $\mathrm{F}$ & $\mathrm{Y}$ & $\mathrm{D}$ & $\mathrm{Y}$ & $\mathrm{E}$ & $\mathrm{V}$ & $\mathrm{E}$ & $\mathrm{R}$ & $\mathrm{I}$ & $\mathrm{I}$ & $\mathrm{N}$ & $\mathrm{II}$ & $\mathrm{P}$ & $\mathrm{D}$ & $\mathrm{Y}$ & $\mathrm{D}$ & $\mathrm{D}$ & $\mathrm{P}$ & $\mathrm{I}$ & $\mathrm{II}$ \\
\hline
\end{tabular}

991 AGCACGACAT CACCGTACTT GTGCTCAAGC GACCCGTCCG CTACAACGTG TACGTGCAGC CTGCCTGTCT GCCCGCGCCT GACGACAACT \begin{tabular}{lllllllllllllllllllllllllllllll}
$\mathrm{E}$ & $\mathrm{H}$ & (D) & $\mathrm{I}$ & $\mathrm{T}$ & $\mathrm{V}$ & $\mathrm{L}$ & $\mathrm{V}$ & $\mathrm{L}$ & $\mathrm{K}$ & $\mathrm{R}$ & $\mathrm{P}$ & $\mathrm{V}$ & $\mathrm{R}$ & $\mathrm{Y}$ & $\mathrm{N}$ & $\mathrm{V}$ & $\mathrm{Y}$ & $\mathrm{V}$ & $\mathrm{Q}$ & $\mathrm{P}$ & $\mathrm{A}$ & $\mathrm{C}$ & $\mathrm{L}$ & $\mathrm{P}$ & $\mathrm{A}$ & $\mathrm{P}$ & $\mathrm{D}$ & $\mathrm{D}$ & $\mathrm{N}$ \\
\hline
\end{tabular}

1081 ACAACAATAT GACTGCTGTC GTAGTCGgTT GGGGAAAGT GGATTTTGGA GGAAGATCGA GCTCAGTTCT ACGAGAAGTG CACGTCCCGG $\begin{array}{lllllllllllllllllllllllllllllllllll} & \mathrm{N} & \mathrm{N} & \mathrm{N} & \mathrm{M} & \mathrm{T} & \mathrm{A} & \mathrm{V} & \mathrm{V} & \mathrm{V} & \mathrm{G} & \mathrm{W} & \mathrm{G} & \mathrm{K} & \mathrm{L} & \mathrm{D} & \mathrm{F} & \mathrm{G} & \mathrm{G} & \mathrm{R} & \mathrm{S} & \mathrm{S} & \mathrm{S} & \mathrm{V} & \mathrm{L} & \mathrm{R} & \mathrm{E} & \mathrm{V} & \mathrm{H} & \mathrm{V} & \mathrm{P}\end{array}$

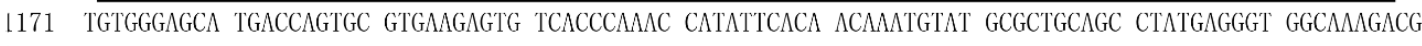
\begin{tabular}{lllllllllllllllllllllllllllllll}
$\mathrm{V}$ & $\mathrm{W}$ & $\mathrm{E}$ & $\mathrm{H}$ & $\mathrm{D}$ & $\mathrm{Q}$ & $\mathrm{C}$ & $\mathrm{V}$ & $\mathrm{K}$ & $\mathrm{S}$ & $\mathrm{V}$ & $\mathrm{T}$ & $\mathrm{Q}$ & $\mathrm{T}$ & $\mathrm{I}$ & $\mathrm{F}$ & $\mathrm{T}$ & $\mathrm{T}$ & $\mathrm{N}$ & $\mathrm{V}$ & $\mathrm{C}$ & $\mathrm{A}$ & $\mathrm{A}$ & $\mathrm{A}$ & $\mathrm{Y}$ & $\mathrm{E}$ & $\mathrm{G}$ & $\mathrm{G}$ & $\mathrm{K}$ & $\mathrm{D}$ \\
\hline
\end{tabular}

1261 CTTGTCAGGg CGACTCGGGA GGTCCTCTGG TTGTACAGAG AGATGACGGC CGCTGGAAGT TGATAGGGGT GGTCTCATGG GGAGTTCAGT \begin{tabular}{lllllllllllllllllllllllllllllllll}
$\mathrm{A}$ & $\mathrm{C}$ & $\mathrm{Q}$ & $\mathrm{G}$ & $\mathrm{D}$ & $\mathrm{S}$ & $\mathrm{G}$ & $\mathrm{G}$ & $\mathrm{G}$ & $\mathrm{P}$ & $\mathrm{I}$ & $\mathrm{V}$ & $\mathrm{V}$ & $\mathrm{Q}$ & $\mathrm{R}$ & $\mathrm{D}$ & $\mathrm{D}$ & $\mathrm{G}$ & $\mathrm{R}$ & $\mathrm{W}$ & $\mathrm{K}$ & $\mathrm{I}$ & $\mathrm{T}$ & $\mathrm{G}$ & $\mathrm{V}$ & $\mathrm{V}$ & $\mathrm{S}$ & $\mathrm{W}$ & $\mathrm{G}$ & $\mathrm{V}$ & $\mathrm{Q}$ \\
\hline
\end{tabular}

1351 GTGCCCTCCC CGGCAAGCCT GGCATTTACA CGGCGGTCAA TAAGTACCTC GATTGGATGA GCGAAGTCAC TGGTGCCTAG tcacaatcat

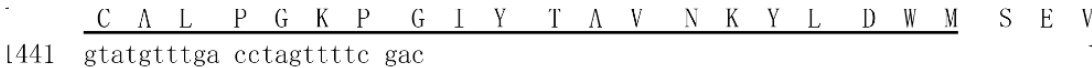

Figure 1. Nucleotide and deduced amino acid sequences of NIPCE3. The start codon (ATG) and stop codon (TAG) are displayed in bold. The numbers on the left side of the figure correspond to the nucleotide sequence. NIPCE3 is composed of a signal peptide (in shaded), a clip domain (dot-lined) with six cysteine (in box), and a trypsin-like serine proteinase domain (underlined). The catalytic triad is marked with a circle $(\mathrm{H}, \mathrm{D}$, and $\mathrm{S})$. The template sequence for in vitro dsNIPCE3 synthesis was pointed (black arrow).

\subsection{Developmental and Tissue-specific Expression of NIPCE3}

qPCR is used to detect the developmental and tissue-specific expression of NIPCE3 (Figure 2). NIPCE3 was found expressed in all developmental stages. Its expression in male adults was significantly higher than in nymphs and female adults (Figure 2A). The expression of NIPCE3 in the gut, ovary, and fat body of adult females was also detected, and the expression was higher in the gut and fat body compared with the ovary (Figure 2B). 
A

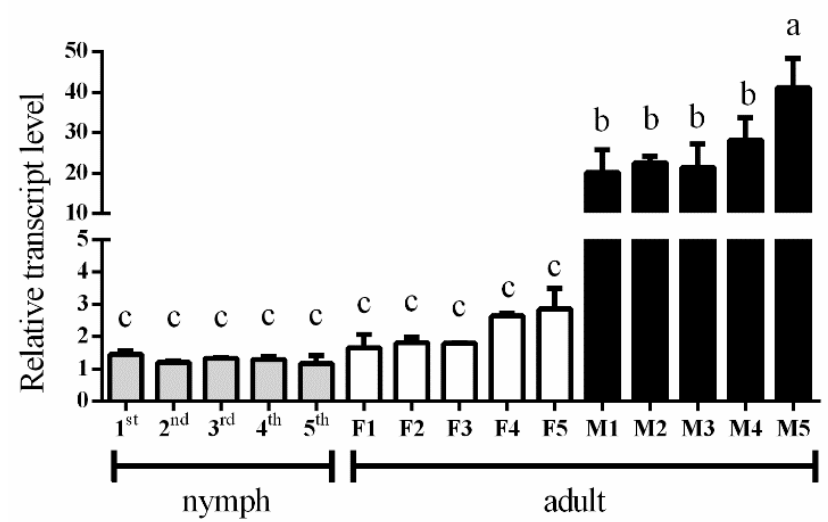

\section{B}

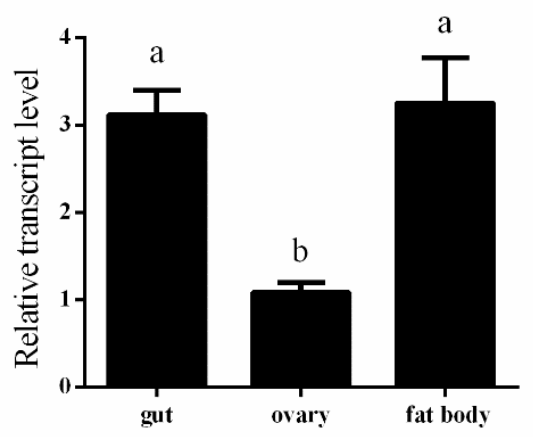

Figure 2. Expression of NIPCE3 in different developmental stages and tissues. (A) Expression patterns of NIPCE3 in different developmental stages, including $1^{\text {st }}$ to $5^{\text {th }}$ nymphs, adults of $\mathrm{F}$ (one- to five-day-old macropterous females) and $\mathrm{M}$ (one- to five-day-old macropterous males). (B) Expression patterns of NIPCE3 in the gut, ovary, and fat body of macropterous females. Data are presented as the mean \pm standard error. Different lower-case letters above the bars indicate significant differences (one way ANOVA was performed using GraphPad Prism Software 6.0, $p<0.05$ ).

\subsection{Effects of RNA Interference}

The expression of NIPCE3 in whole body was notably inhibited two days (96.7\%), three days (97.5\%) and five days (95.6\%) after NIPCE3 dsRNA (dsNIPCE3) injection, compared with GFP dsRNA (dsGFP) injection (Figure 3A), indicating that dsNIPCE3 effectively inhibits the expression of NIPCE3.

Eggs were detained in follicles after dsNIPCE3 injection, while some eggs of dsGFP-treated individuals have been ovulated (Figure 3B). On the contrary, only a few to no eggs were laid by females after dsNIPCE3 treatment, whereas each female laid more than 150 eggs on average after dsGFP treatment (Figure 3C), indicating that dsNIPCE3 affects the oviposition of N. lugens.

Mortality following dsRNA treatment was also calculated (Figure 3D). The result showed that dsNIPCE3-treated females died at a higher rate than dsGFP-treated females, although the difference was not statistically significant. The difference in mortality between dsNIPCE3-treated and dsGFP-treated largely occurred during the first five days.

Ovarian development was affected by dsNIPCE3 injection. Up to four days after dsRNA injection (immature stage of the ovary), most ovaries were not visibly different between dsNIPCE3 and dsGFP injection under anatomic microscope, but from the $5^{\text {th }}$ day after dsRNA injection a difference was observable. At the $5^{\text {th }}$ day after dsRNA injection, in the dsGFP group, the terminal follicles in ovarioles presented a number of banana-shaped mature eggs (Figure 4A,C), but in the dsNIPCE3 treatment group, malformed pear-shaped or spherical terminal follicles were observed (Figure 4B). The egg in the terminal follicles were not typically banana-shaped. The posterior part of the egg was spherical and its eggshell was not formed while at the anterior its eggshell was formed, or below the center the egg was expanded and the diameter was much bigger than the normal egg (Figure 4D,E). These results suggest that eggshell is not normally formed after dsNIPCE3 injection. 
A

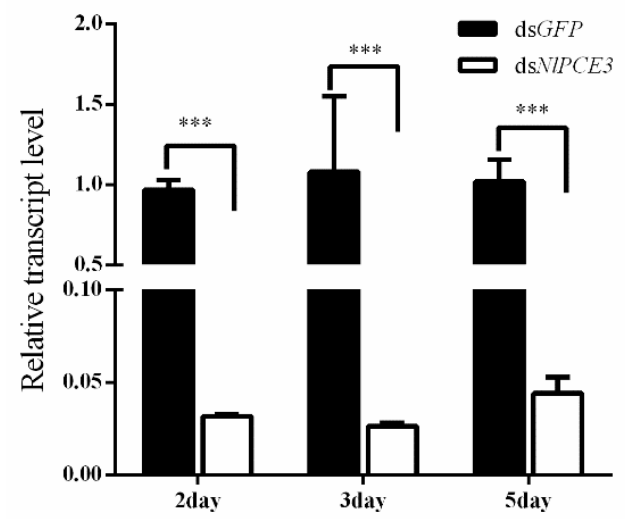

C

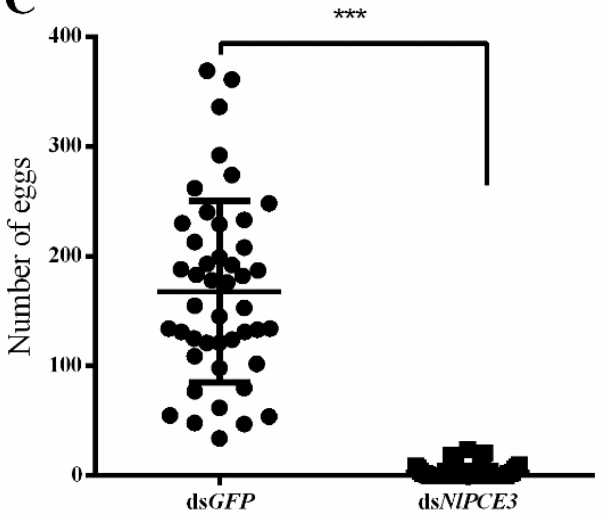

B

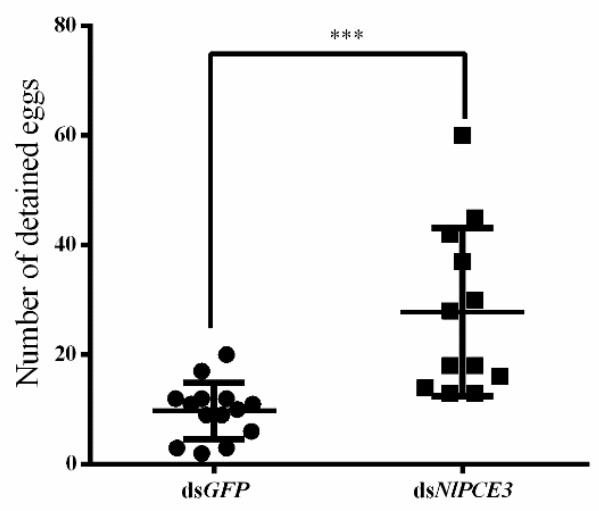

D

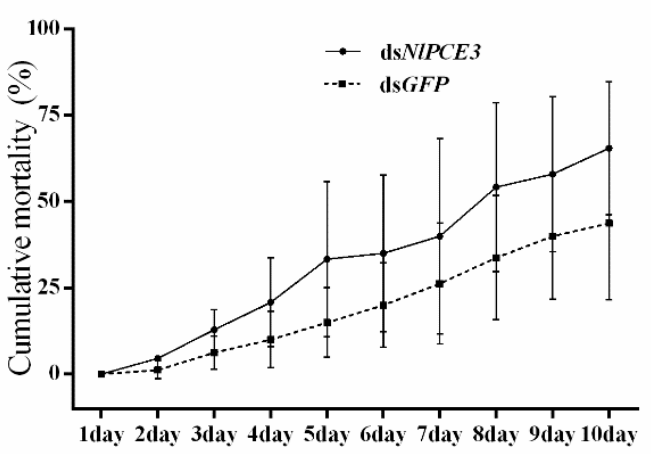

Figure 3. The effects of RNA interference. (A) Down-regulation of NIPCE3 using dsNIPCE3 in different days. (B) The number of detained eggs in female adults at $5^{\text {th }}$ day after injected with dsNIPCE3 $(\mathrm{n}=12)$ or dsGFP $(\mathrm{n}=14)$. (C) The number of eggs laid by females injected with dsNIPCE3 $(\mathrm{n}=55)$ or dsGFP $(\mathrm{n}$ = 45). (D) The cumulative mortality (\%) of N. lugens after dsNIPCE3 and dsGFP injection (three groups, 20 individuals in each group). All data are presented as the mean \pm standard error, one way ANOVA and unpaired two-tailed Student's t-tests were performed using GraphPad Prism Software 6.0. ${ }^{* * *}$ in (A-C) represents 0.001 significance of difference. No significant difference was found in (D). 

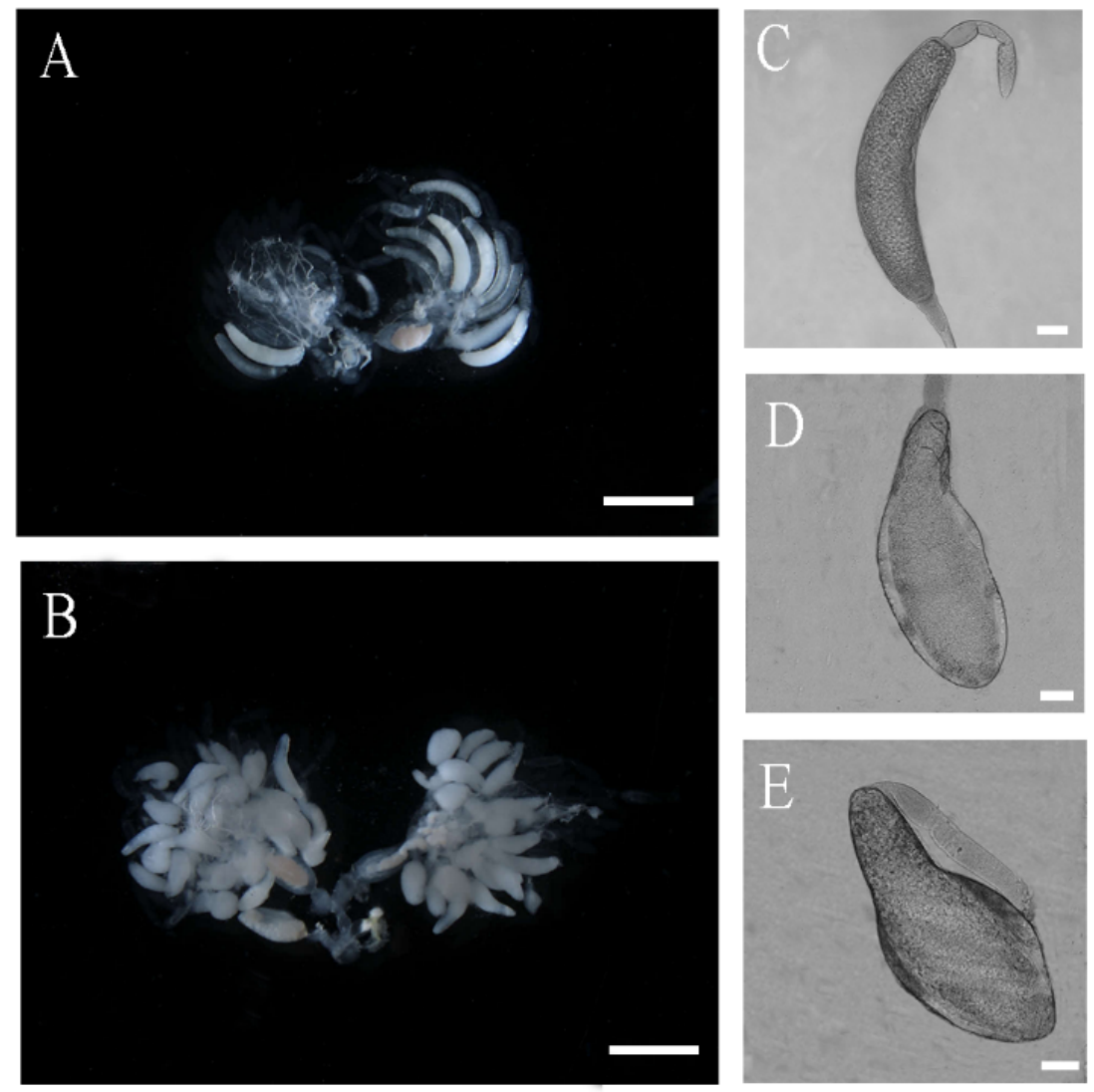

Figure 4. Effect of dsNIPCE3 on Nilaparvata lugens ovarian development. (A) Ovaries of a control female adult treated at $5^{\text {th }}$ day after dsGFP injection. (B) Ovaries of a female adult at $5^{\text {th }}$ day after dsNlPCE3 injection. (C) Normal mature egg. (D-E) Abnormal egg. Scale bar: $1 \mathrm{~mm}(\mathbf{A}, \mathbf{B}) ; 100 \mu \mathrm{m}(\mathbf{C}-\mathbf{E})$.

\subsection{Immunofluorescence Analysis of NIPCE3 Expression}

To better understand the functions of NlPCE3, we performed immunofluorescence staining. NlPCE3 expression was observed in fat body (Figure 5 A,B), gut (Figure 5C-F), and ovary (Figure 5G,H). After dsNIPCE3 treatment, no phenotypical change of the fat body or gut was observed. In detail, in gut epithelial cells, NIPCE3 was expressed longitudinally, relative to the axis of the gut. In the ovary, NIPCE3 was predominantly expressed in the cytoplasm and cytomembrane of follicular cells outside oocytes (Figures 5H and 6D-F), and its expression appeared to be up-regulated with the onset of vitellogenesis (Figure 5H1-H4). Starting from the $5^{\text {th }}$ day after dsNIPCE3 injection, the follicular cells outside the terminal follicle were destroyed, and the expression of NLPCE3 in follicular cells was disordered (Figure 6A-C). 

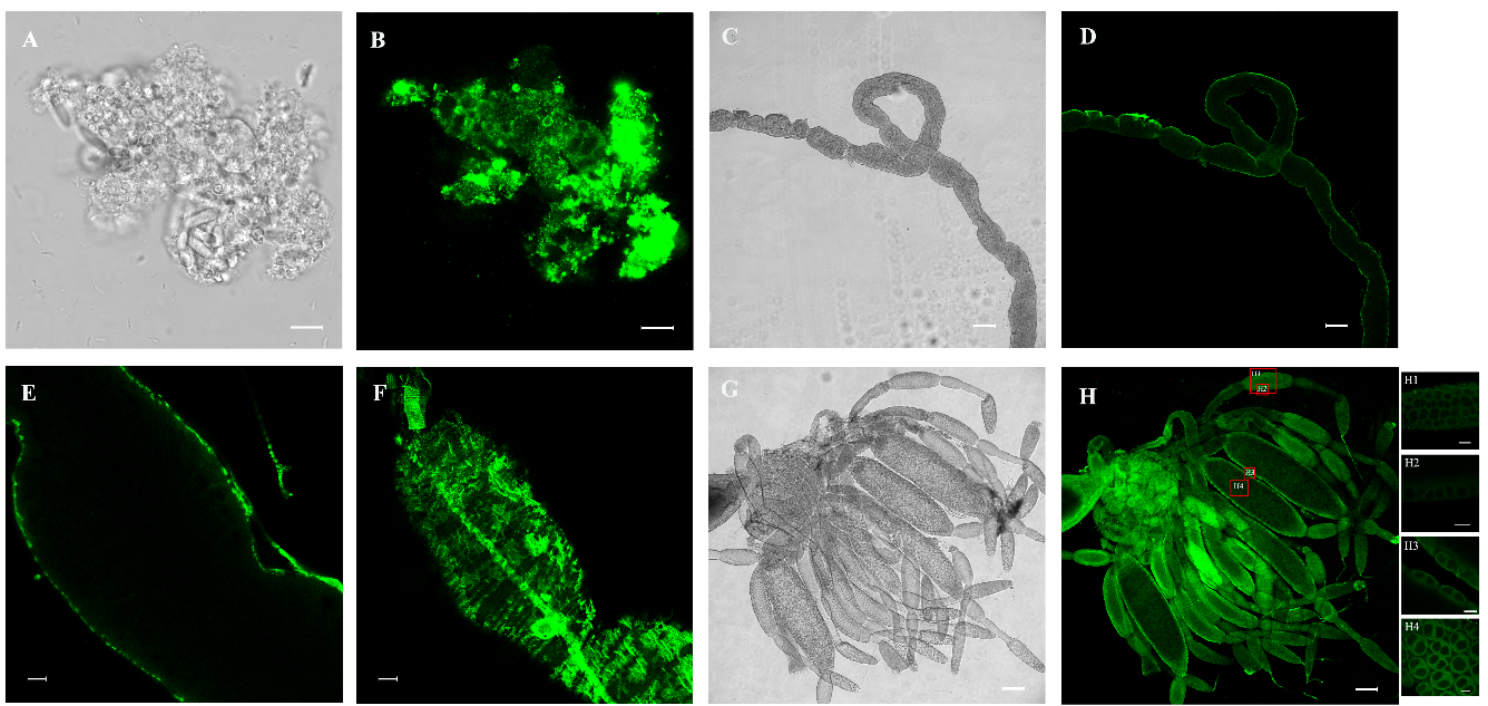

Figure 5. Analysis of NIPCE3 expression using immunofluorescence staining. (A,B) The localization of NlPCE3 in fat body. (C-F) The localization of NIPCE3 in gut. (D-H) The localization of NlPCE3 in ovary. $\mathrm{H} 1$ to $\mathrm{H} 4$ were respectively enlarged from $\mathrm{H}$ (red boxes). Scale bar: $10 \mu \mathrm{m}$ (A,B,E,F,H1-H4); 100 $\mu \mathrm{m}(\mathbf{C}, \mathbf{D}, \mathbf{G}, \mathbf{H})$.
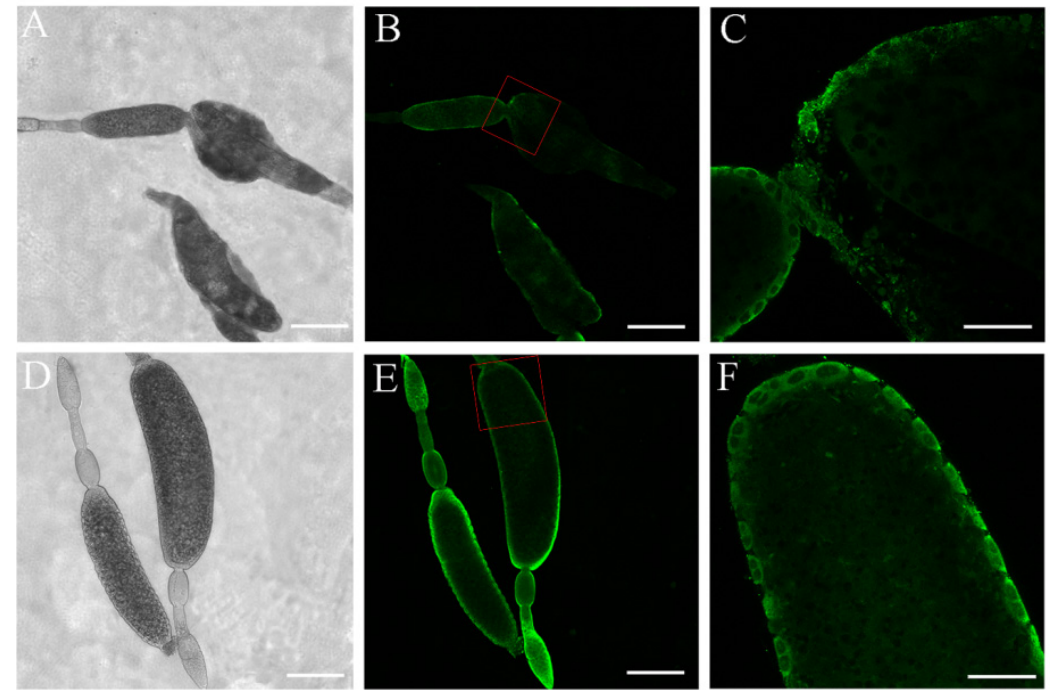

Figure 6. Ovarian follicle development after dsRNA treatment. (A,B) Follicles of females at $5^{\text {th }}$ day after dsNIPCE3 treatment. (C) Enlarged from B (red box). (D-E) Follicles of females at $5^{\text {th }}$ day after dsGFP treatment. (F) Enlarged from D (red box). Scale bar: $200 \mu \mathrm{m}(\mathbf{A}, \mathbf{B}, \mathbf{D}, \mathbf{E}) ; 50 \mu \mathrm{m}(\mathbf{C}, \mathbf{F})$.

\section{Discussion}

In the present study, NIPCE3 is expressed at all developmental stages and in different tissues of $N$. lugens, indicating that NIPCE3 ubiquitously functions in N. lugens. Immunofluorescence staining results also show that NIPCE3 is expressed in fat body and gut, but no obvious phenotypical change was found after dsNIPCE3 injection. These results might be due to the followed reasons. Firstly, there might be a difference in RNAi efficiency, related to tissue-specific effort or the way of RNAi (injection or feeding). Secondly, no phenotypical change exerted after dsRNA injection in fat body and gut was possible due to compensatory/rescue mechanisms, as the NIPCE3 expression was relatively higher in fat body and gut than ovary. Alternatively, the phenotypical changes were inconspicuous and require other technologies to explore. 
The effect of NIPCE3 RNAi on ovarian development was prominent. After treatment with dsNIPCE3, the follicular cells underwent degradation without clear boundaries between cells and could not maintain a regular cell layer outside terminal oocytes. Notably, NIPCE3 RNAi caused the abnormal development of terminal follicles, the abnormal eggshell formation of terminal oocytes, and the obstruction of ovulation. These findings indicate that NIPCE3 is necessary for normal egg development and ovulation.

Ovarial follicular cells function in patterning the oocyte, physically shaping the egg chamber, and secreting the eggshell [29]. Went and Junquera [30] demonstrated that the shape of the follicle in Heteropeza pygmaea was maintained by its follicular cells. In D. melanogaster and Bradysia tritici, the basement membrane of the follicular epithelium is an important factor in shaping the follicles, since cells tend to assume a spherical shape in the absence of external factors (e.g., substrate or adhesive neighboring cells) or internal forces (e.g., cytoskeleton) [31]. As reported recently, in D. melanogaster, the follicular cells secrete an extracellular matrix that may transform the egg chamber from spherical to elliptical [32]. In the present study, NIPCE3 is widely expressed in the follicular cells. Besides, degradation of follicular cells outside the malformed terminal oocyte was found after NIPCE3 RNAi. Degradation of follicular cells usually occurs in intact insects after movement of eggs into the lateral oviduct, but in the present study, the degradation of follicular cells occurred before the eggshell of the terminal oocytes was completely formed. Therefore, NIPCE3 RNAi disturbed the structure intactness and function of follicular cells, and then resulted in abnormal formation of the eggshell of the terminal oocytes.

The result that dsNIPCE3-treated females laid much fewer eggs than dsGFP-treated females in this study may be due to three reasons. Firstly, because the follicular cells outside the terminal oocyte were destroyed, these cells could not support the egg to move down into the lateral oviduct of the ovary. Secondly, the abnormal eggs had a large morphology compared with the banana-shaped egg, which may arise the obstruction of ovulation. Thirdly, after treatment with dsNIPCE3, ovarioles were brittle in dissection, indicating the strength of the ovariole might not support the egg to move from the terminal follicle to the lateral oviduct.

Egg production is a complex activity affected by multiple factors, and some studies have tried to explore the underlined mechanism of follicular cells functioning in the process of egg production. During oogenesis of Blattella germanica, a calcium-binding glycoprotein, SPARC, contributes to oogenesis in panoistic ovaries by stabilizing the follicular cells program, helping to maintain the nuclear division and the cytoskeleton organization [33]. In N. lugens, Dicer1 helps the follicular cells providing nutrients for oocytes, and Dicer1 dsRNA treatment causes abnormal follicular cells and smaller, badly malformed oocytes [34]. In another study in N. lugens, the depletion of forkhead box transcription factor L2 (NIFoxL2) or follicle cell protein 3C (NIFcp3C), which is directly activated by NIFoxL2, suppresses the expression of genes encoding high-cysteine chorion proteins. Follicular cells can then not form proper chorion layers, resulting in spherically-shaped oocytes, obesity, and female infertility [35]. This is similar to the results of dsNIPCE 3 treatment in the present study. However, the malformation of follicles and eggs in the present study may be ascribed to the degradation of follicular cells rather than the chorion layer. Since NIPCE3 has a signal peptide, it could be secreted out the follicular cells, but the result of immunofluorescence staining showed NIPCE3 was predominantly expressed in the cytoplasm and cytomembrane of follicular cells. Therefore, NIPCE3 possibly remains inside these cells and in membranes contributing to maintaining the stability of the follicular cells. Besides, the clip domain may have a regulatory function or may modulate interactions of proteases with their substrates or other proteins [12]. NIPCE3 may therefore function in egg production by cascade regulation or covalent modification, which warrants further study.

\section{Conclusions}

In conclusion, functional analysis by RNAi revealed that NIPCE3 influences the eggshell formation of eggs and ovulation, indicating that NlPCE3 plays an important role in egg production in N. lugens. 
This is an important addition to the other known functions of clip-SPs. In this study, NIPCE3 has significantly higher expression in male adults than in female adults, in accord with the previous study [5]. Additionally, NIPCE3 is expressed higher in the gut and fat body, but no obvious phenotypical change was observed in these tissues after RNAi. These results need further studies to explain.

Supplementary Materials: The following are available online at http:/www.mdpi.com/2075-4450/10/11/378/s1, Figure S1: Specificity of anti-NlPCE3 antibody analysed by western blot, Figure S2: Amino acid sequence comparison of clip-SPs, Figure S3: Amino acid sequence comparison of NIPCE1-5.

Author Contributions: Conceptualization, J.-m.W. and Y.-p.X.; investigation, J.-m.W., R.-e.Z., R.-j.Z. and J.-1.J.; validation, J.-m.W.; supervision, Y.-p.X. and X.-p.Y.; writing-original draft, J.-m.W.; writing—review and editing, Y.-p.X. and X.-p.Y.

Funding: This work was supported by grants from the National Natural Science Foundation of China (31871961 and 31501632) and a grant from the Zhejiang Provincial Key R\&D Project (2019C02015).

Conflicts of Interest: The authors declare no conflicts of interest.

\section{References}

1. Jena, K.K.; Kim, S.M. Current status of brown planthopper (BPH) resistance and genetics. Rice 2010, 3, 161-171. [CrossRef]

2. Mao, K.; Zhang, X.; Ali, E.; Liao, X.; Jin, R.; Ren, Z.; Wan, H.; Li, J. Characterization of nitenpyram resistance in Nilaparvata lugens (Stål). Pestic. Biochem. Physiol. 2019, 157, 26-32. [CrossRef] [PubMed]

3. Lu, K.; Shu, Y.; Zhou, J.; Zhang, X.; Chen, M.; Yao, Q.; Zhou, Q.; Zhang, W. Molecular characterization and RNA interference analysis of vitellogenin receptor from Nilaparvata lugens (Stål). J. Insect Physiol. 2015, 73, 20-29. [CrossRef] [PubMed]

4. Zhang, B.X.; Huang, H.J.; Yu, B.; Lou, Y.H.; Fan, H.W.; Zhang, C.X. Bicaudal-C plays a vital role in oogenesis in Nilaparvata lugens (Hemiptera: Delphacidae). J. Insect Physiol. 2015, 79, 19-26. [CrossRef] [PubMed]

5. Bao, Y.Y.; Qu, L.Y.; Zhao, D.; Chen, L.B.; Jin, H.Y.; Xu, L.M.; Cheng, J.A.; Zhang, C.X. The genome- and transcriptome-wide analysis of innate immunity in the brown planthopper, Nilaparvata lugens. BMC Genomics 2013, 14, 160. [CrossRef] [PubMed]

6. Zhang, D.; Wan, W.; Kong, T.; Zhang, M.; Aweya, J.J.; Gong, Y.; Li, S. A clip domain serine protease regulates the expression of proPO and hemolymph clotting in mud crab, Scylla paramamosain. Fish Shellfish Immunol. 2018, 79, 52-64. [CrossRef]

7. Jiang, H.; Kanost, M.R. The clip-domain family of serine proteinases in arthropods. Insect Biochem. Mol. Biol. 2000, 30, 95-105. [CrossRef]

8. Muta, T.; Hashimoto, R.; Miyata, T.; Nishimura, H.; Toh, Y.; Iwanaga, S. Proclotting enzyme from horseshoe crab hemocytes: cDNA cloning, disulfide locations, and subcellular localization. J. Biol. Chem. 1990, 265, 22426-22433.

9. Iwanaka, S. Biochemical principle of Limulus test for detecting bacterial endotoxins. Proc. Jpn. Acad. Ser. B Phys. Biol. Sci. 2007, 83, 110-119. [CrossRef]

10. Zhu, L.; Song, L.; Zhao, J.; Xu, W.; Chang, Y. Molecular cloning, characterization and expression of a serine protease with clip-domain homologue from scallop Chlamys farreri. Fish Shellfish Immunol. 2007, 22, 556-566. [CrossRef]

11. Amparyup, P.; Wiriyaukaradecha, K.; Charoensapsri, W.; Tassanakajon, A. A clip domain serine proteinase plays a role in antibacterial defense but is not required for prophenoloxidase activation in shrimp. Dev. Comp. Immunol. 2010, 34, 168-176. [CrossRef] [PubMed]

12. Jiang, H.; Wang, Y.; Kanost, M.R. Pro-phenol oxidase activating proteinase from an insect, Manduca sexta: A bacteria-inducible protein similar to Drosophila easter. Proc. Natl. Acad. Sci. USA 1998, 95, 12220-12225. [CrossRef] [PubMed]

13. Jiang, H.; Wang, Y.; Yu, X.Q.; Zhu, Y.; Kanost, M. Prophenoloxidase-activating proteinase-3 (PAP-3) from Manduca sexta hemolymph: A clip-domain serine proteinase regulated by serpin-1J and serine proteinase homologs. Insect Biochem. Mol. Biol. 2003, 33, 1049-1060. [CrossRef]

14. Gupta, S.; Wang, Y.; Jiang, H. Manduca sexta prophenoloxidase (proPO) activation requires proPO-activating proteinase (PAP) and serine proteinase homologs (SPHs) simultaneously. Insect Biochem. Mol. Biol. 2005, 35, 241-248. [CrossRef] [PubMed] 
15. Zou, Z.; Shin, S.W.; Alvarez, K.S.; Kokoza, V.; Raikhel, A.S. Distinct melanization pathways in the mosquito Aedes aegypti. Immunity 2010, 32, 41-53. [CrossRef]

16. Barillas-Mury, C. CLIP proteases and plasmodium melanization in Anopheles gambiae. Trends Parasitol. 2007, 23, 297-299. [CrossRef]

17. Volz, J.; Osta, M.A.; Kafatos, F.C.; Muller, H.M. The roles of two clip domain serine proteases in innate immune responses of the malaria vector Anopheles gambiae. J. Biol. Chem. 2005, 280, 40161-40168. [CrossRef]

18. Kan, H.; Kim, C.H.; Kwon, H.M.; Park, J.W.; Roh, K.B.; Lee, H.; Park, B.J.; Zhang, R.; Zhang, J.; Soderhall, K.; et al. Molecular control of phenoloxidase-induced melanin synthesis in an insect. J. Biol. Chem. 2008, 283, 25316-25323. [CrossRef]

19. Kwon, T.H.; Kim, M.S.; Choi, H.W.; Joo, C.H.; Cho, M.Y.; Lee, B.L. A masquerade-like serine proteinase homologue is necessary for phenoloxidase activity in the coleopteran insect, Holotrichia diomphalia larvae. Eur. J. Biochem. 2000, 267, 6188-6196. [CrossRef]

20. Lee, S.Y.; Cho, M.Y.; Hyun, J.H.; Lee, K.M.; Homma, K.I.; Natori, S.; Kawabata, S.I.; Iwanaga, S.; Lee, B.L. Molecular cloning of cDNA for pro-phenol-oxidase-activating factor I, a serine protease is induced by lipopolysaccharide or 1,3-beta-glucan in coleopteran insect, Holotrichia diomphalia larvae. Eur. J. Biochem. 1998, 257, 615-621. [CrossRef]

21. Satoh, D.; Horii, A.; Ochiai, M.; Ashida, M. Prophenoloxidase-activating enzyme of the silkworm, Bombyx mori. Purification, characterization, and cDNA cloning. J. Biol. Chem. 1999, 274, 7441-7453. [CrossRef] [PubMed]

22. Jang, I.; Nam, H.; Lee, W. CLIP-domain serine proteases in Drosophila innate immunity. BMB Rep. 2008, 41, 102-107. [CrossRef] [PubMed]

23. Kanost, M.R.; Jiang, H. Clip-domain serine proteases as immune factors in insect hemolymph. Curr. Opin. Insect Sci. 2015, 11, 47-55. [CrossRef] [PubMed]

24. Anderson, K.V. Pinning down positional information: Dorsal-ventral polarity in the Drosophila embryo. Cell 1998, 95, 439-442. [CrossRef]

25. Kanost, M.R.; Clem, R.J. Insect proteases. In Insect Molecular Biology and Biochemistry; Gilbert, I.L., Ed.; Academic Press: San Diego, CA, USA, 2012; Volume 10, pp. 346-364. [CrossRef]

26. Bao, Y.Y.; Qin, X.; Yu, B.; Chen, L.B.; Wang, Z.C.; Zhang, C.X. Genomic insights into the serine protease gene family and expression profile analysis in the planthopper, Nilaparvata lugens. BMC Genomics 2014, 15, 507. [CrossRef]

27. Milligan, J.F.; Groebe, D.R.; Witherell, G.W.; Unlenbeck, O.C. Oligoribonucleotide synthesis using T7 RNA polymerase and synthetic DNA templates. Nucleic Acids Res. 1987, 15, 8783-8798. [CrossRef]

28. Nan, G.H.; Xu, Y.P.; Yu, Y.W.; Zhao, C.X.; Zhang, C.X.; Yu, X.P. Oocyte vitellogenesis triggers the entry of yeast-like symbionts into the oocyte of brown planthopper (Hemiptera: Delphacidae). Ann. Entomol. Soc. Am. 2016, 109, 753-758. [CrossRef]

29. Horne-Badovinac, S.; Bilder, D. Mass transit: epithelial morphogenesis in the Drosophila egg chamber. Dev. Dyn. 2005, 232, 559-574. [CrossRef]

30. Went, D.F.; Junquera, P. Embryonic development of insect eggs formed without follicular epithelium. Dev. Biol. 1981, 86, 100-110. [CrossRef]

31. Gutzeit, H.O.; Haas-Assenbaum, A. The somatic envelopes around the germ-line cells of polytrophic insect follicles: Structural and functional aspects. Tissue Cell 1991, 23, 853-865. [CrossRef]

32. Heifetz, Y.; Tram, U. Oogenesis, final oocyte maturation and ovulation, Insects. In Encyclopedia of Reproduction, 2nd ed.; Skinner, K.M., Ed.; Academic Press: San Diego, CA, USA, 2018; Volume 6, pp. 239-245.

33. Irles, P.; Ramos, S.; Piulachs, M.D. SPARC preserves follicular epithelium integrity in insect ovaries. Dev. Biol. 2017, 422, 105-114. [CrossRef] [PubMed]

34. Zhang, X.; Lu, K.; Zhou, Q. Dicer1 is crucial for the oocyte maturation of telotrophic ovary in Nilaparvata lugens (Stål) (Hemiptera: Geometroidea). Arch. Insect Biochem. Physiol. 2013, 84, 194-208. [CrossRef] [PubMed]

35. Ye, Y.X.; Pan, P.L.; Xu, J.Y.; Shen, Z.F.; Kang, D.; Lu, J.B.; Hu, Q.L.; Huang, H.J.; Lou, Y.H.; Zhou, N.M.; et al. Forkhead box transcription factor L2 activates Fcp3C to regulate insect chorion formation. Open Biol. 2017, 7, 170061. [CrossRef] [PubMed]

(C) 2019 by the authors. Licensee MDPI, Basel, Switzerland. This article is an open access article distributed under the terms and conditions of the Creative Commons Attribution (CC BY) license (http://creativecommons.org/licenses/by/4.0/). 